\title{
GMTIFS: challenging optical design problems and their solutions for the GMT integral-field spectrograph
}

\author{
John Hart, Gabe Bloxham, Robert Boz, Brady Espeland, Robert Sharp \\ Research School of Astronomy and Astrophysics, College of Physical and Mathematical Sciences, \\ The Australian National University, Cotter Road, Weston, ACT, 2611, Australia
}

\begin{abstract}
GMTIFS is a first generation instrument for the Giant Magellan Telescope (GMT). It is a combined Imager and Integral Field Spectrograph (IFS) designed to work with the Adaptive Optics (AO) Systems of the GMT. Working at the diffraction limit of the GMT and satisfying the challenging AO interface requirements and constraints results in unique optical challenges. We describe two of these challenges and how we have addressed them. The GMT has a direct feed architecture that maximizes transmission and reduces emissivity. This means that the cryostat window is tilted to reflect visual wavelengths to the external Visual Wave Front Subsystem (VWS). For a plane-parallel window, this tilt causes astigmatism in the transmitted beam that must be corrected. A corrective system using two plates, tilted and slightly wedged in opposite directions, is used. Geometry and performance of the system is described. Another challenging problem is the optical design of the anamorphic field projector. The Integral Field Unit of GMTIFS requires that a small field delivered to it be projected onto an Image Slicer at much larger scale, with the magnification in the spectral direction being twice that in the spatial direction so that the spaxels are square when referred to the sky. Output images must be coincident in the spectral and spatial projections in both the field and pupil domains. Additionally, field and pupil image locations must be independently controllable so that they can be made coincident for interchangeable units that provide a range of output field scales. A two-mirror system that satisfies these requirements is described.
\end{abstract}

Keywords: GMT, cryostat window, Imager, Integral Field Spectrograph, anamorphic distortion, square spaxels.

\section{INTRODUCTION}

GMTIFS is a diffraction limited GMT instrument that includes a multi-scale Integral Field Spectrograph (IFS), an Imager, and an On-Instrument Wave Front Sensor (OIWFS). A conceptual design description was given in a 2012 SPIE paper $^{1}$, when it was one of six potential first-light instruments for GMT, after which it was down-selected for further development. It operates over the $Y, J, H$ and $K$-bands. The instrument accepts a $180 \operatorname{arcsec}(180 \mathrm{~mm})$ diameter field. A star image in all but the central $20 \times 20$ arcsec science field can be steered to the OIWFS. The central science field is picked-off and delivered to the Imager, having 5 mas pixels on a $4 \mathrm{~K}$ square detector. Dichroic beam splitting is used within the science field to feed the IFS, offering four anamorphically distorted scales with square spaxels of $6,12,25$ and 50 mas. Corresponding fields are $0.28 \times 0.56,0.56 \times 1.11,1.11 \times 2.23$ and $2.23 \times 4.46$ arcsec sliced into 45 slitlets. Spectral resolving powers of 5000 and 10000 are available on a $4 \mathrm{~K}$ square detector.

GMTIFS presents many challenging optical design problems. Two examples are described here.

\subsection{Cryostat Window}

The cryostat window passes a $180 \mathrm{~mm}$ diameter field into the cryostat. The window is tilted by 20 deg so that it can also act as a dichroic beam splitter to deliver a reflected image to the external Visual Wave Front Subsystem (VWS). A window in the form of a single plane parallel plate is not acceptable because the tilt generates excessive aberration (mostly astigmatism) in the transmitted beam. A corrected two-element window system has therefore been devised to

\footnotetext{
${ }^{1}$ http://adsabs.harvard.edu/cgi-bin/nphdata_query?bibcode=2012SPIE.8446E..1IM\&db_key=AST\&link_type=ABSTRACT\&high=568ca950e931914
} Proc. of SPIE Vol. 9908, 99089F - (c) 2016 SPIE · CCC code: 0277-786X/16/\$18 · doi: 10.1117/12.2231552 
correct the transmitted aberration. It is also arranged to provide adequately small angular deviation of the transmitted beam, and thereby avoid significant decenter of the pupil image. The quality of the beam transmitted into the cryostat is defined here. The quality of the beam reflected to the external wave front sensor system is also important, but is not discussed here beyond noting that the window is made thick enough to adequately control aberration due to deformation under atmospheric pressure loading.

\subsection{Anamorphic Field Projector}

A range of four differently sized field images enter the IFS through a set of interchangeable focal plane masks, and must be projected to the image slicer at a much larger fixed size. A set of projectors (relays with high magnification) is therefore required between the focal plane masks and the image slicer. These projectors are made anamorphic (providing different magnifications in the spectral and spatial directions) as a means of providing square spaxels when referred to the sky.

Such anamorphic projectors require at least two separated optical elements with particular anamorphic constraint to maintain coincident spectral and spatial focus (avoid astigmatism). Further anamorphic constraint is required to achieve the same property for pupil imagery. Yet further constraint is then needed to make both field and pupil imagery parfocal for the range of projector sets. Two-element systems have enough degrees of freedom to satisfy all of these conditions.

\section{CRYOSTAT WINDOW}

\subsection{Description}

Good aberration correction can be achieved by using two slightly wedged plates tilted in opposite directions. The wedge angle and thickness can both be different for the two plates. For the particular arrangement chosen here, the geometry is constrained so that the wedge angles of the two plates are the same (to minimize cost), but oriented in opposite directions. This is shown in Figure 1. For the small wedge angles needed to correct the field image, the net angular deviation of the optical axis is small relative to wedge angle. There is then very little displacement of, or chromatic aberration in, the pupil image. Importantly, there is also no significant tilt of the focal plane with respect to the optical axis.

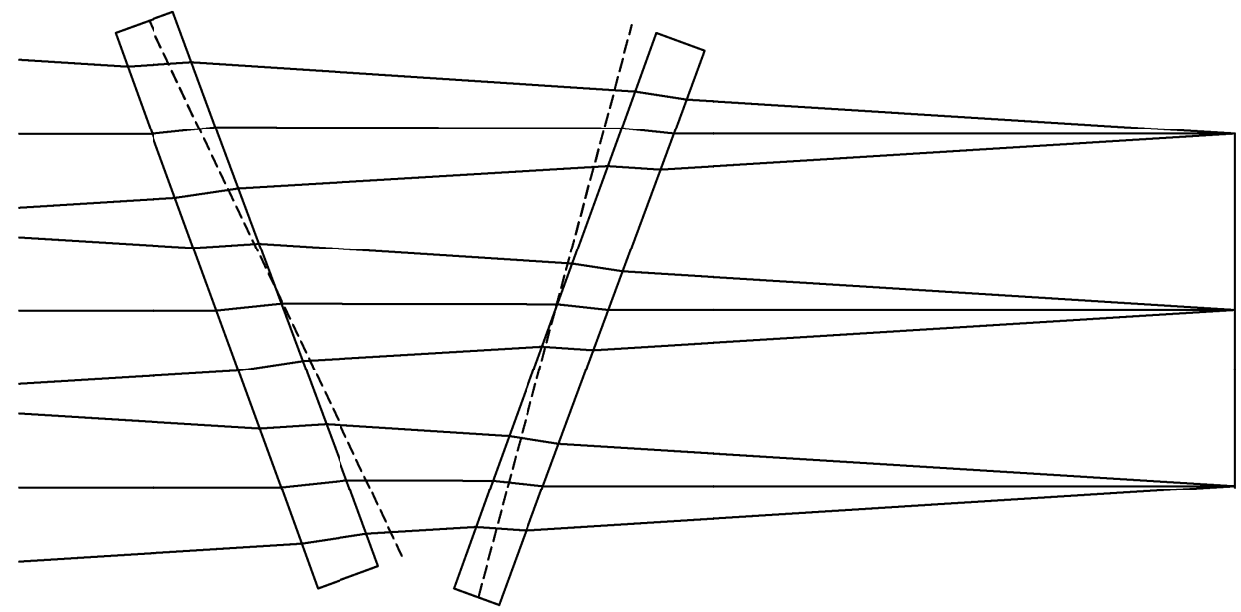

Figure 1: Layout of the corrected beam splitter window system.

Astigmatism correction is achieved by using two flat and slightly wedged plates. Dashed lines indicate wedging in exaggerated form.

The slight wedging is not obvious in the correctly scaled (solid lines) view, but its direction is indicated in the exaggerated view (dashed lines). Any error in wedge angle matching can be corrected by tilt adjustment of the downstream plate.

An appealing option is to set the tilt of the second plate so that telecentric chief rays at a central design wavelength are un-deviated. Thus, there is no pupil decenter at the chosen wavelength, and decenter at adjacent wavelengths is minimal. 
Lateral color varies linearly across the field, passing through zero at a position where the separation between the two plates has a particular value. Ideally, this location would be set to the center of the field in order to deliver the best possible image quality to the $20 \mathrm{~mm}$ square field of the science instrument, but this is not possible with the zerodeviation condition.

The zero-deviation condition is therefore abandoned in favor of annulling lateral color in the center of the field so that the science instruments benefit. The consequent pupil decenter is defined in $\$ 2.4$.

Performance is optimized by adjusting the common wedge angle of the two plates, the tilt of the second plate and the thickness of the second plate. The thickness of the first plate (which bears the pressure load and carries the dichroic coating) is determined as the value for which pressure distortion maximizes correction of the telescope astigmatism over the field (it acts partially as a reflective Gascoigne corrector). The distance from the first plate to the input focal plane is set to $500 \mathrm{~mm}$, as required by the VWS. The material is chosen to have low refractive index and dispersion.

The specifications so derived are listed in Table 1.

Table 1: Window system specifications.

\begin{tabular}{|c|c|c|c|c|c|c|c|}
\hline $\begin{array}{l}\text { Field } \\
\text { Diameter } \\
\text { (mm or arcsec) }\end{array}$ & $\begin{array}{c}1 \text { st } \\
\text { Front } \\
\text { Face } \\
\text { Tilt } \\
(\operatorname{deg}) \\
\end{array}$ & $\begin{array}{c}\text { 2nd } \\
\text { Front } \\
\text { Face } \\
\text { Tilt } \\
\text { (deg) }\end{array}$ & $\begin{array}{l}\text { Wedge } \\
\text { Angle } \\
\text { (deg) }\end{array}$ & $\begin{array}{c}\text { 1st } \\
\text { Plate } \\
\text { Thickness } \\
\text { (mm) }\end{array}$ & $\begin{array}{c}\text { 2nd } \\
\text { Plate } \\
\text { Thickness } \\
\text { (mm) }\end{array}$ & $\begin{array}{l}\text { Plate } \\
\text { Separation } \\
(\mathrm{mm})\end{array}$ & Material \\
\hline 180 & 20 & -20.150416 & \pm 0.322776 & 32 & 26.011410 & 140 & Calcium Fluoride \\
\hline
\end{tabular}

Plate thickness and plate separation are as measured without face tilts or wedge. Wedge angle is established by tilting the second surface about its local center. Front face tilt is established by tilting the whole plate about the center of the front face.

\subsection{Field Imagery}

The ratio of RMS wave front error $\sigma$ to wavelength $\lambda$ for the window is shown in Figure 2 for the whole wavelength range at the center and edges of the $180 \mathrm{~mm}$ diameter field. These three field positions are indicated by the height $y$ from the field center. The curve labeled $y=0$ is more or less applicable to the $20 \mathrm{~mm}$ square science field located in the center of the OIWFS field.



Figure 2: Wave front error versus wavelength for the full wavelength range at three representative field positions.

The dominant aberration away from the minima is defocus caused by longitudinal color. Focus is set to give the same value at the ends of the wavelength range for the science field curve $(y=0)$. 
The three curves of Figure 2 are re-plotted in Figure 3, where the three field positions are shown in separate panels, and additional features are included.

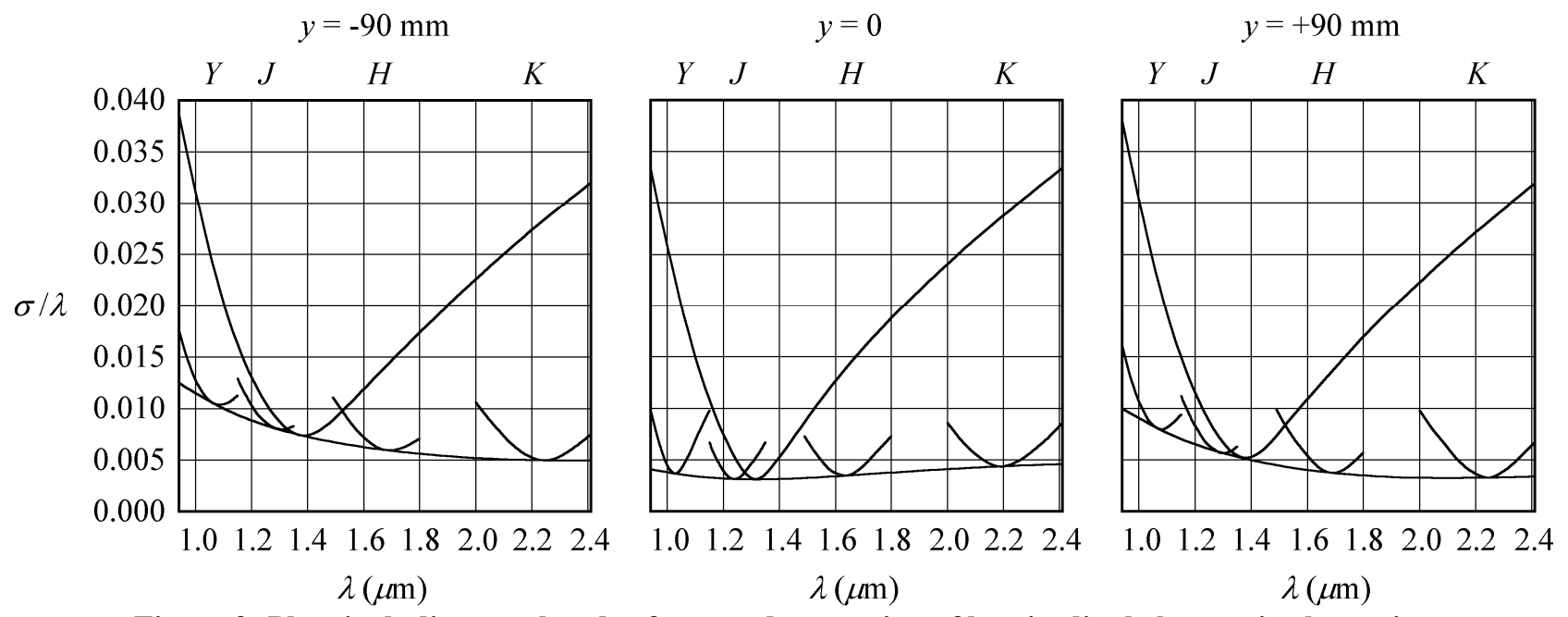

Figure 3: Plots including passband refocus and correction of longitudinal chromatic aberration.

The four short curves shown in each panel represent the $Y, J, H$ and $K$ band segments of the full curves, refocused for end-to-end balance in the science field $(y=0)$. The underlying envelope curves are tangent to all these curves, and represent window performance when longitudinal color is corrected by downstream optics.

As can be seen, the color-corrected aberration for the science field $(y=0)$ is then

$$
\frac{\sigma}{\lambda}<0.005
$$

This can be compared to the benchmark value corresponding to the diffraction limit. Defining the diffraction limit as having a Strehl ratio of $S=0.8$, it is

$$
\begin{aligned}
\frac{\sigma}{\lambda} & =\frac{\sqrt{-\ln S}}{2 \pi} \\
& =0.075
\end{aligned}
$$

Thus measured, window performance is excellent.

The foregoing wave front error analysis accounts for longitudinal color, but not for lateral color. Lateral color is accounted for separately, as shown in Figure 4. It is measured as the distance $\delta y$ between centroids of images at maximum and minimum wavelengths of the indicated pass bands. 


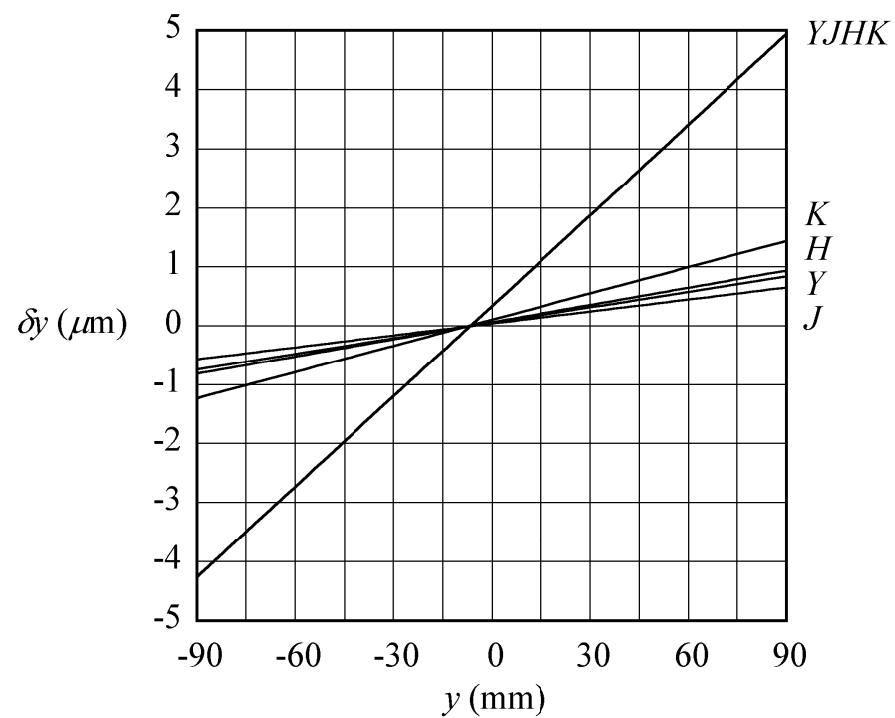

Figure 4: Lateral color versus field position for all pass bands.

The aberration is distributed linearly with respect to field position, and is arranged to be zero at (or at least near) $y=0$. This minimizes lateral color within the centrally located $20 \mathrm{~mm}$ square science field.

Assuming that all the curves of Figure 4 pass through $\delta y=0$ at $y=0$, the range of $\delta y$ across the science field for the $Y, J$, $H$ and $K$ bands are, respectively, $0.176,0.136,0.195$ and $0.297 \mu \mathrm{m} \mathrm{PV}$. These values correspond to $0.051,0.039,0.056$ and $0.086 \mu \mathrm{m}$ RMS ( $2 \sqrt{3}$ times less).

These image aberrations are roughly converted to an equivalent wave front error by assuming a smooth error form (astigmatism). The central wavelengths $\lambda$ of the $Y, J, H$ and $K$ bands are $1.05,1.25,1.65$ and $2.2 \mu \mathrm{m}$ respectively. For a focal ratio $F=8$,

$$
\frac{\sigma}{\lambda}=\frac{1}{4 \sqrt{3}} \frac{\delta y}{F}
$$

Numerical values are shown in Table 2.

Table 2: Equivalent wave front error for lateral color in the $\mathbf{2 0} \mathbf{~ m m}$ square science field.

\begin{tabular}{|l|c|c|c|c|}
\hline Band & $Y$ & $J$ & $H$ & $K$ \\
\hline$\sigma / \lambda$ & 0.0009 & 0.0006 & 0.0006 & 0.0007 \\
\hline
\end{tabular}

These are an order of magnitude smaller than the values shown for the science field in Figure 3, and are therefore not significant.

The window also causes some slight anamorphic distortion of the field imagery, this being the inverse of the pupil image distortion factors shown in $\$ 2.3$. This is of no importance.

\subsection{Pupil Distortion}

Assuming telecentric feed, the only significant pupil image aberration generated by the window is anamorphic distortion that is effectively independent of wavelength, as investigated here.

The pupil is imaged through the $20 \mathrm{~mm}$ square science field mask, and the diffraction limit imposed by this is the reference for assessing the image quality. For a telescope aperture diameter $d=25 \mathrm{~m}$, focal ratio $F=8$, science aperture width $a=20 \mathrm{~mm}$ and wavelength $\lambda$ ranging from 0.94 to $2.42 \mu \mathrm{m}$, the diffraction limit (Rayleigh criterion) referred to the telescope aperture is:

$$
\begin{aligned}
\frac{d}{a} F \lambda & =10000 \lambda \\
& =9-24 \mathrm{~mm}
\end{aligned}
$$


Off-axis rays in the plane perpendicular to the window tilt are un-deviated. Those in the plane of the window tilt are deviated in proportion to their off-axis angle, thus causing anamorphic pupil distortion. From ray tracing, the distortion factors in this direction are $1.00328,1.00326$ and 1.00323 for wavelengths of $0.94,1.65$ and $2.42 \mu \mathrm{m}$ respectively. Referred to the $25 \mathrm{~m}$ telescope aperture diameter, the corresponding increases in pupil diameter in the tilt direction are $82.0,81.5$ and $80.8 \mathrm{~mm}$ respectively. The maximum displacement of image points from their best-fit circularly symmetric pattern is therefore about $20 \mathrm{~mm}$. This limits the masking accuracy of the cold stop to a level that is comparable to the diffraction limit.

\subsection{Pupil Decenter}

Angular deviation of the chief rays is a measure of pupil decenter. It is determined as follows.

For the first plate, with front face tilt $\alpha_{1}$, wedge angle $A_{1}$ and refractive index $n_{1}$, the angular deviation is

$$
\varepsilon_{1}=\alpha_{1}-A_{1}-\arcsin \left(\sin \alpha_{1} \cos A_{1}-\sin A_{1} \sqrt{n_{1}^{2}-\sin ^{2} \alpha_{1}}\right)
$$

For the second plate, with front face tilt $\alpha_{2}$, wedge angle $A_{2}$ and refractive index $n_{2}$, the angular deviation is

$$
\varepsilon_{2}=\left(\alpha_{2}+\varepsilon_{1}\right)-A_{2}-\arcsin \left(\sin \left(\alpha_{2}+\varepsilon_{1}\right) \cos A_{2}-\sin A_{2} \sqrt{n_{2}{ }^{2}-\sin ^{2}\left(\alpha_{2}+\varepsilon_{1}\right)}\right)
$$

The combined angular deviation is

$$
\varepsilon=\varepsilon_{1}+\varepsilon_{2}
$$

Pupil decenter $e$ is conveniently expressed in units of pupil diameter $d$. For a focal ratio $F=8$ of the window beam,

$$
\frac{e}{d}=\varepsilon F
$$

This is plotted against wavelength in Figure 5.

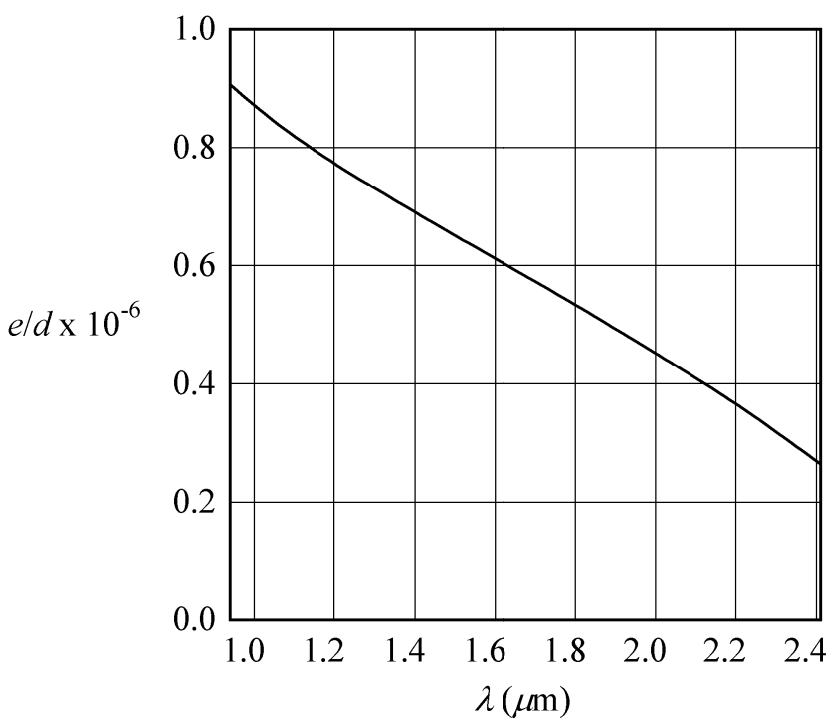

Figure 5: The ratio of pupil decenter $e$ to pupil diameter $d$, plotted against wavelength.

As can be seen, $e / d$ is small $\left(<1 \times 10^{-6}\right)$ over the whole wavelength range. Zero deviation occurs at a wavelength of 2.93 $\mu \mathrm{m}$. 


\section{ANAMORPHIC FIELD PROJECTOR}

\subsection{Description}

A reflective Anamorphic Field Projector complying with the requirements described in $\S 1.2$ is shown in Figure 6 . The mirrors are toroids having different curvatures in the spectral (vertical) and spatial (horizontal) projections. They are tilted as needed to avoid obscuration. The input field is at the left. The anamorphically distorted output beam (which therefore has an elliptical cross-section) is shown truncated on its way to the distant image slicer, and is almost collimated because the magnification is high (and different in the spectral and spatial projections).

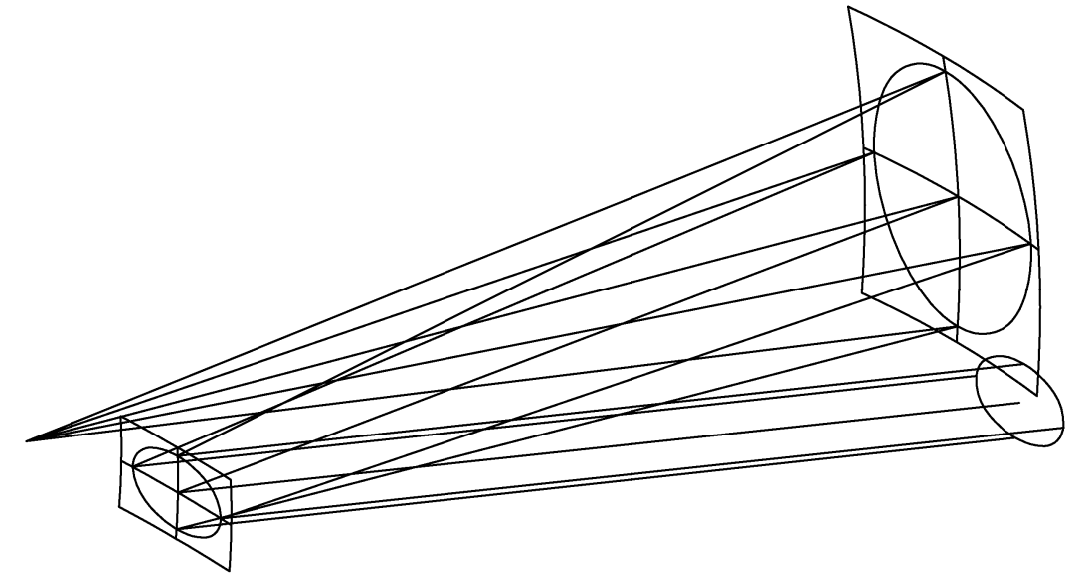

Figure 6: A pair of toric mirrors arranged as an Anamorphic Field Projector.

The required geometry is defined as follows.

\subsection{Anastigmatic Field and Pupil Imagery}

Field and pupil image geometry are shown for one of the two anamorphic projections in Figure 7, without the mirror tilt needed to avoid obscuration. The output field imagery beam (top view) is almost collimated because the magnification is high. The input pupil imagery beam (bottom view) is likewise almost collimated because the telescope output is almost telecentric. True collimation is assumed in both modes for this analysis.

Where relevant in the analysis, the nomenclature shown is distinguished for the spectral and spatial projections by applying subscripts $x$ and $y$ respectively. 


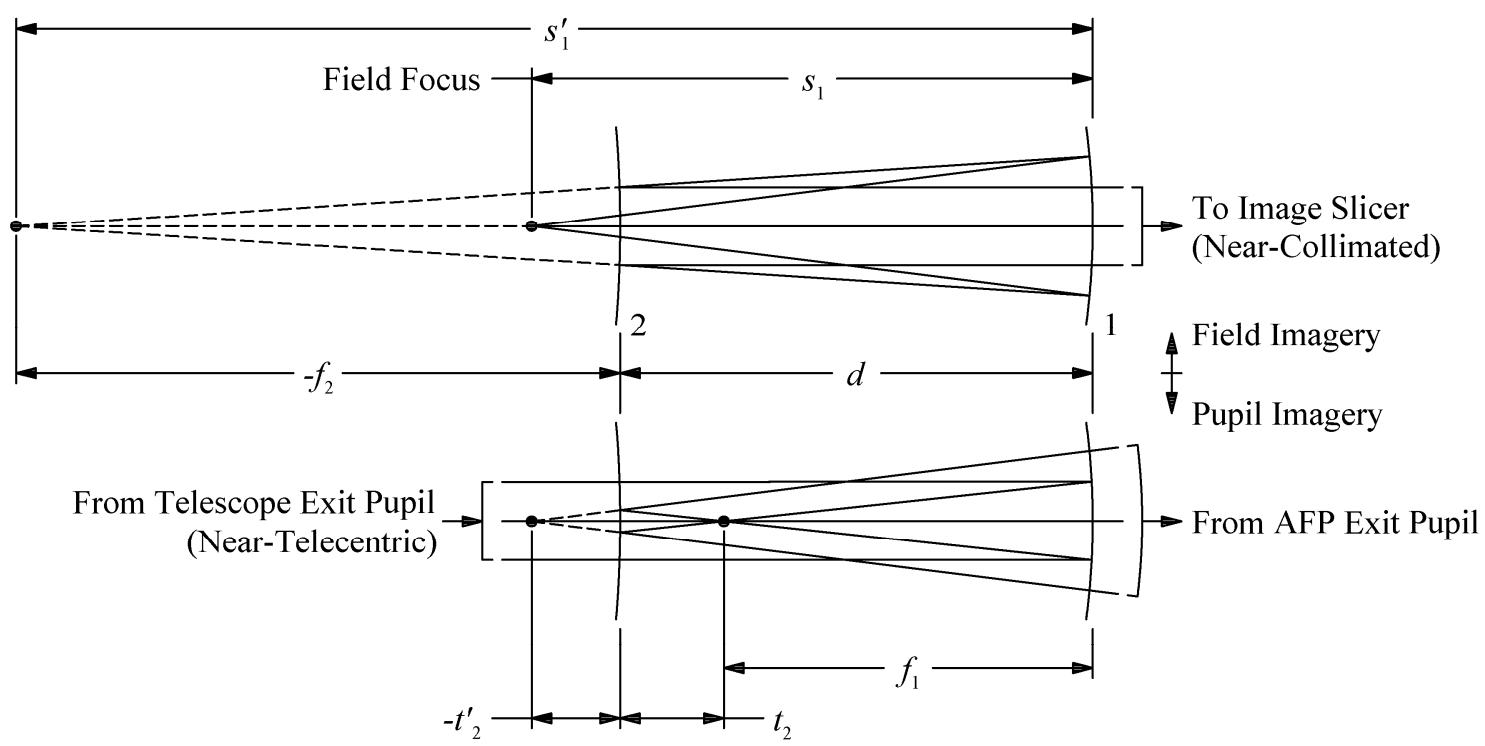

Figure 7: Field and pupil image geometry for one anamorphic projection.

For field and pupil imagery respectively,

$$
\begin{array}{cc}
s_{1}^{\prime}=d-f_{2} & t_{2}=d-f_{1} \\
\frac{1}{f_{1}}=\frac{1}{s_{1}}+\frac{1}{s_{1}^{\prime}} & \frac{1}{f_{2}}=\frac{1}{t_{2}}+\frac{1}{t_{2}^{\prime}} \\
s_{1}=\frac{f_{1}\left(f_{2}-d\right)}{f_{1}+f_{2}-d} & t_{2}^{\prime}=\frac{f_{2}\left(f_{1}-d\right)}{f_{1}+f_{2}-d}
\end{array}
$$

The distance between the entry field and exit pupil is

$$
u=s_{1}+t_{2}^{\prime}-d
$$

For anastigmatic spectral and spatial foci respectively,

$$
\begin{aligned}
s_{1 y}=s_{1 x} & t_{2 y}^{\prime}=t_{2 x}^{\prime} \\
\frac{f_{1 x}+f_{2 x}-d}{f_{1 y}+f_{2 y}+d}=\frac{f_{1 x}\left(f_{2 x}-d\right)}{f_{1 y}\left(f_{2 y}-d\right)} & \frac{f_{1 x}+f_{2 x}-d}{f_{1 y}+f_{2 y}+d}=\frac{f_{2 x}\left(f_{1 x}-d\right)}{f_{2 y}\left(f_{1 y}-d\right)}
\end{aligned}
$$

The combined focal lengths in the spectral and spatial projections are, respectively,

$$
f_{x}=\frac{f_{1 x} f_{2 x}}{f_{1 x}+f_{2 x}-d} \quad f_{y}=\frac{f_{1 y} f_{2 y}}{f_{1 y}+f_{2 y}-d}
$$


The anamorphic distortion factor is

$$
\begin{aligned}
M & =\frac{f_{y}}{f_{x}} \\
& =\frac{f_{2 y}\left(f_{2 x}-d\right)}{f_{2 x}\left(f_{2 y}-d\right)} \\
& =\frac{f_{1 y}\left(f_{1 x}-d\right)}{f_{1 x}\left(f_{1 y}-d\right)}
\end{aligned}
$$

Combining the forgoing equations yields

$$
\begin{gathered}
\frac{d}{f_{1 y}}=\frac{d / f_{1 x}-1+M}{M} \\
\frac{d}{f_{2 x}}=\frac{d / f_{1 x}-1-M}{d / f_{1 x}-1} \\
\frac{d}{f_{2 y}}=\frac{d / f_{1 x}-2}{d / f_{1 x}-1} \\
\frac{f_{x}}{d}=\frac{1}{M+1} \\
\frac{f_{y}}{d}=\frac{M}{M+1} \\
\frac{s_{1}}{d}=\frac{M}{(M+1)\left(d / f_{1 x}-1\right)} \\
\frac{t_{2}^{\prime}}{d}=\frac{1-d / f_{1 x}}{M+1} \\
\frac{\left(d / f_{1 x}\right)^{2}+(M-1) d / f_{1 x}-2 M}{(M+1)\left(1-d / f_{1 x}\right)}
\end{gathered}
$$

In the absence of anamorphic distortion at the diffraction gratings (Littrow configuration), the need for square spaxels on the sky requires the anamorphic distortion factor to be $M= \pm 2$. Those of the foregoing functions that vary with $d / f_{1 x}$ are plotted in Figure 8 for $\mathrm{M}=-2$ and Figure 9 for $\mathrm{M}=+2$. 

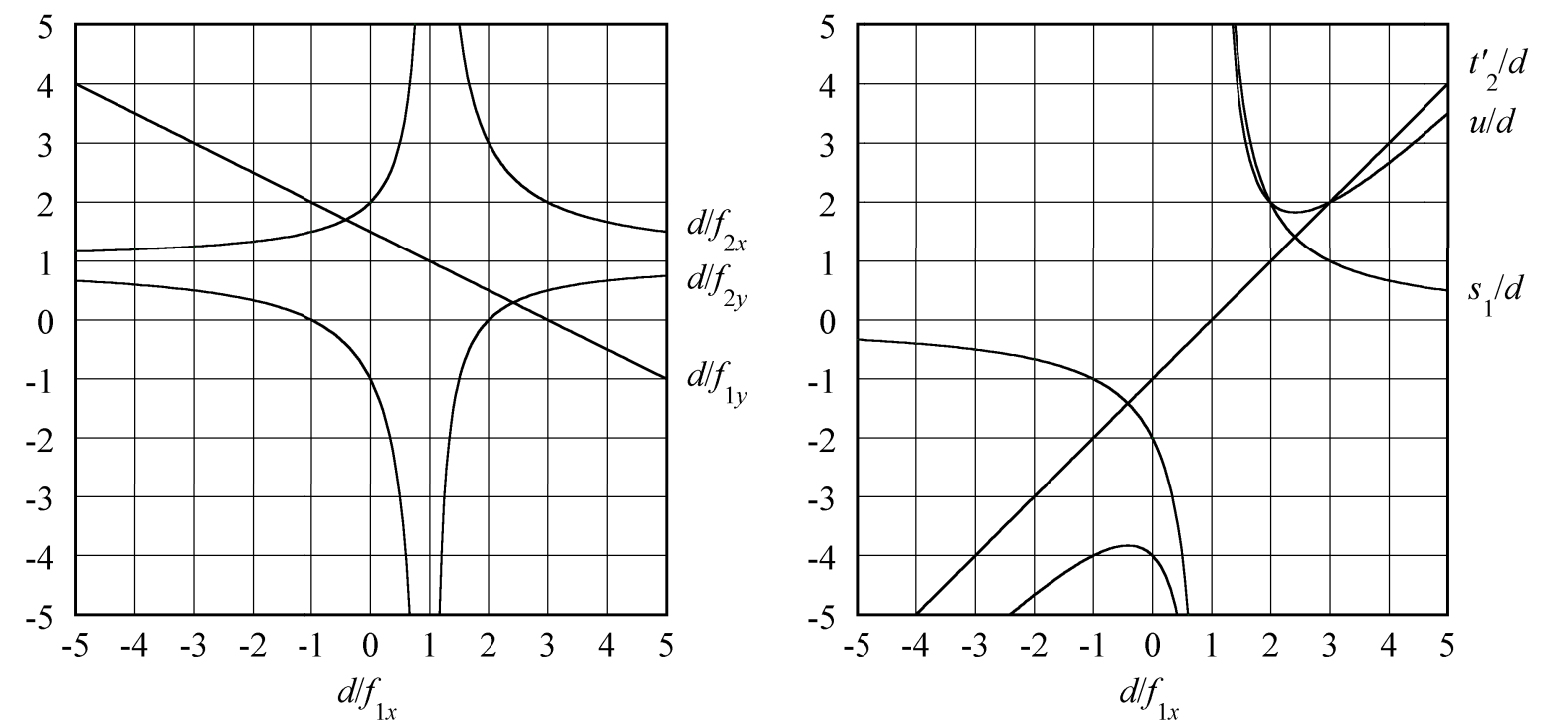

Figure 8: Plots of functions that vary with $f_{1 x}$ for $M=-2$
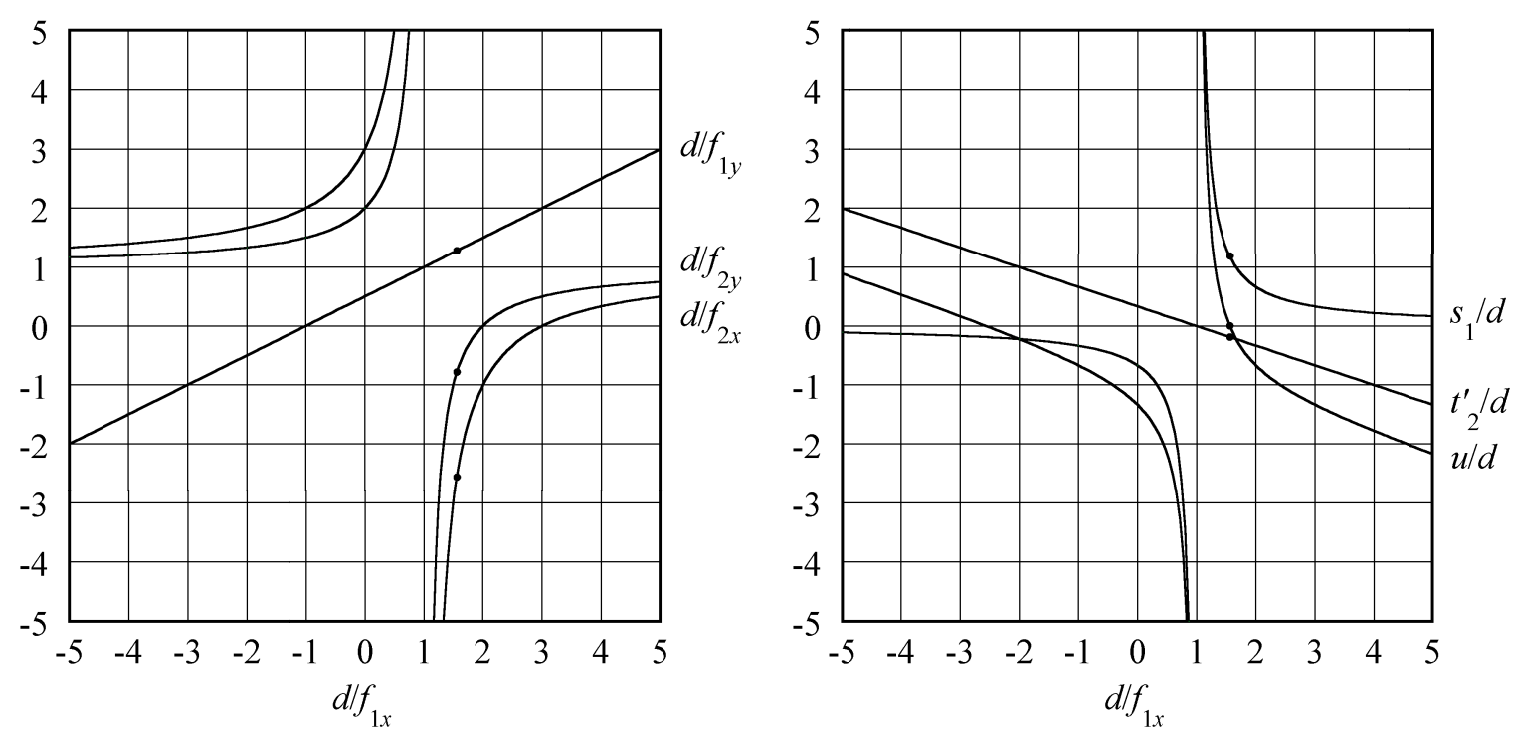

Figure 9: Plots of functions that vary with $f_{1 x}$ for $M=+2$

It can be seen from these plots that there are always two projector configurations that have the same value of $u / d$, this being a measure of the longitudinal distance between the input field and the exit pupil. In fact, these configurations are the same, except that the mirrors are interchanged. Effectively, the same system can be run forwards or backwards.

The marker dots shown in Figure 9 indicate the selected condition were the input field and the exit pupil are longitudinally coincident $(u=0)$, as discussed in $\S 3.3$. 


\subsection{Exit Pupil Location}

If a range of projectors is used to provide different image scales, they should all share the same exit pupil location. That is, the distance $u$ from the input field to the exit pupil should not vary. Re-forming the last equation of $\$ 3.2$ so that this distance can be independently specified gives

$$
\frac{d}{f_{1 x}}=\frac{d(1-M)-u(1+M) \pm \sqrt{d^{2}\left(1+6 M+M^{2}\right)+u^{2}\left(1+2 M+M^{2}\right)+u\left(2 d+4 M d+2 M^{2} d\right)}}{2 d}
$$

For $M=-2$

$$
\frac{d}{f_{1 x}}=\frac{3 d+u \pm \sqrt{-7 d^{2}+u^{2}+2 d} u}{2 d}
$$

For $\mathrm{M}=+2$

$$
\frac{d}{f_{1 x}}=\frac{-d-3 u \pm \sqrt{17 d^{2}+9 u^{2}+18 d u}}{2 d}
$$

Clearly, there is a solution (real roots) for all values of $u$ when $M$ is positive, but not when $M$ is negative.

An appealing option is to set $u=0$, so that the exit pupil is coincident with the input field. The exit pupil location is then the same for geometrically similar projectors of different scale. As can be seen from Figure 8 and Figure 9, this can only be achieved for $M=+2$ (the roots are imaginary for $M=-2$ ). For this case,

$$
\frac{d}{f_{1 x}}=\frac{-1 \pm \sqrt{17}}{2}
$$

Additionally,

$$
\begin{gathered}
\frac{d}{f_{1 y}}=\frac{1 \pm \sqrt{17}}{4} \\
\frac{d}{f_{2 x}}=\frac{-7 \pm \sqrt{17}}{-3 \pm \sqrt{17}} \\
\frac{d}{f_{2 y}}=\frac{-5 \pm \sqrt{17}}{-3 \pm \sqrt{17}} \\
\frac{f_{x}}{d}=\frac{1}{3} \\
\frac{f_{y}}{d}=\frac{2}{3} \\
\frac{s_{1}}{d}=\frac{4}{3(-3 \pm \sqrt{17})} \\
\frac{t_{2}^{\prime}}{d}=\frac{3 \mp \sqrt{17}}{6} \\
\frac{u}{d}=0
\end{gathered}
$$


These equations define the shape of the geometrically similar AFP suite, but not the size. The size is defined by the magnification required between the input field focus and the output field at the Image Slicer. This is done by specifying the required mirror separation $d$, as shown in $\S 3.6$.

A graphical interpretation of this pupil location condition is shown for the spectral projection in Figure 10. The extended mirror circles are mutually tangent at the point diametrally opposite the optical vertices.

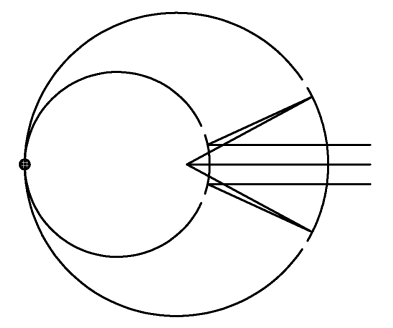

Figure 10: Mutually tangent mirror circles in the spectral projection for $M=+2$ and $u=0$.

Further properties of this condition are that

$$
\begin{gathered}
f_{1 x} f_{2 x}=-\frac{1}{4} d^{2} \\
f_{1 y} f_{2 y}=-d^{2} \\
f_{1 x} f_{1 y}=f_{2 x} f_{2 y}
\end{gathered}
$$

All projectors in a range providing different magnifications are then geometrically similar; they differ only in size, not shape. The scale of the image projected onto the slicer is inversely proportional to this size.

\subsection{Focus Error and Astigmatism Correction}

The spectral and spatial curvatures of the mirrors are independently determined, and error in their values will therefore cause displacements of the output focus that is independent in the two projections. Thus, both focus error and astigmatism can occur as a result of manufacturing error. The former can be corrected by displacing the input image, and the latter by changing the mirror separation, as explained below.

As defined in $\S 3.2$, the distance from the first mirror to the intermediate focus is, in the spectral and spatial projections respectively,

$$
s_{1 x}^{\prime}=d-f_{2 x} \quad s_{1 y}^{\prime}=d-f_{2 y}
$$

Given that output beams are taken to be collimated in this geometry, it is convenient to refer image displacements and astigmatism to the input focus with the output image being fixed (collimated). Then, when the mirror separation is changed by moving the second mirror a small distance $\delta d$, the intermediate image also moves by this distance in both projections. This corresponds to movements of the input image in the spectral and spatial projections of $\delta s_{1 x}$ and $\delta s_{1 y}$ respectively. The ratio of these movements to the change in mirror separation is, respectively,

$$
\frac{\delta s_{1 x}}{\delta d}=\left(\frac{s_{1}}{s_{1 x}^{\prime}}\right)^{2} \quad \frac{\delta s_{1 y}}{\delta d}=\left(\frac{s_{1}}{s_{1 y}^{\prime}}\right)^{2}
$$

Longitudinal astigmatism is the difference $\delta s_{1}$ between the two input image displacements. The ratio of this to the change in mirror separation is

$$
\frac{\delta s_{1}}{\delta d}=\left(\frac{s_{1}}{s_{1 x}^{\prime}}\right)^{2}-\left(\frac{s_{1}}{s_{1 y}^{\prime}}\right)^{2}
$$




\subsection{Input Field Decenter}

The off-axis configuration required to avoid obscuration results in decenter of the input field relative to the exit pupil, as shown in Figure 11.



Figure 11: The AFP viewed in the spectral projection to show the decenter between input image and exit pupil.

The exit pupils are arranged to all be centered on the optical axis to optimize performance of the downstream optics. The input fields are therefore decentered.

A view of the adjacent input images and exit pupils is shown in Figure 12, where all four configurations are overlaid. It shows the actual layout, drawn four times true size. The elliptical exit pupils are all centered on the optical axis. The rectangular input image fields are displaced in proportion to their image scale.



Figure 12: Decentred input fields (rectangular) and centered exit pupils (elliptical) for the AFP suite. Scale 4:1.

The offset field geometry is numerically defined in Table 3 .

Table 3: Offset field dimensions for the four AFP configurations.

\begin{tabular}{|r|r|c|c|c|}
\hline \multicolumn{2}{|l|}{$\begin{array}{l}\text { Slit } \\
\text { Width }\end{array}$} & \multicolumn{3}{|c|}{ AFP Input Field Dimensions } \\
\hline nrad & mas & $\begin{array}{c}\text { Spectral } \\
\text { Width } \\
(\mathrm{mm})\end{array}$ & $\begin{array}{c}\text { Spatial } \\
\text { Width } \\
(\mathrm{mm})\end{array}$ & $\begin{array}{c}\text { Spectral } \\
\text { Offset } \\
(\mathrm{mm})\end{array}$ \\
\hline 30 & 6 & 0.280 & 0.560 & 0.936 \\
\hline 60 & 12 & 0.560 & 1.121 & 1.872 \\
\hline 120 & 25 & 1.121 & 2.242 & 3.740 \\
\hline 240 & 50 & 2.242 & 4.484 & 7.466 \\
\hline
\end{tabular}

\subsection{The AFP Suite}

A suite of projectors is adopted for which the anamorphic distortion factor is $M=+2$ and the longitudinal separation of the input field and exit pupil is $u=0$, as described in $§ 3.3$. All projectors are therefore geometrically similar, varying only in size.

Size is determined by specifying the mirror separation $d$ required to provide the appropriate magnification between the input field focus and the Image Slicer, as follows. 
For a distance between the input field and the image slicer $l=2000 \mathrm{~mm}$, telescope focal length $f_{\text {tel }}=207588.7 \mathrm{~mm}$, image slicer slit width $\delta x=2 \mathrm{~mm}$ and angular slit on the sky $\delta \gamma$, the required focal length of the projector in the spectral direction is

$$
\begin{aligned}
d & =3 f_{x} \\
& =3 \frac{l f_{t e l} \delta \gamma}{\delta x}
\end{aligned}
$$

Using this equation and the foregoing theory, paraxial values of the projector parameters are listed to three decimal places in Table 4 for the range of angular slit widths shown.

\begin{tabular}{|c|c|c|c|c|c|c|c|c|c|c|c|c|c|}
\hline \multicolumn{2}{|l|}{$\delta \gamma$} & \multirow{2}{*}{$\begin{array}{c}d \\
(\mathrm{~mm})\end{array}$} & \multirow{2}{*}{$\begin{array}{c}f_{1 x} \\
(\mathrm{~mm})\end{array}$} & \multirow{2}{*}{$\begin{array}{c}f_{1 y} \\
(\mathrm{~mm})\end{array}$} & \multirow{2}{*}{$\begin{array}{c}f_{2 \mathrm{x}} \\
(\mathrm{mm})\end{array}$} & \multirow{2}{*}{$\begin{array}{c}f_{2 y} \\
(\mathrm{~mm})\end{array}$} & \multirow{2}{*}{$\begin{array}{c}f_{x} \\
(\mathrm{~mm})\end{array}$} & \multirow{2}{*}{$\begin{array}{c}f_{y} \\
(\mathrm{~mm})\end{array}$} & \multirow{2}{*}{$\begin{array}{c}s_{1} \\
(\mathrm{~mm})\end{array}$} & \multirow{2}{*}{$\begin{array}{c}t_{2}^{\prime} \\
(\mathrm{mm})\end{array}$} & \multirow{2}{*}{$\frac{\delta s_{1 x}}{\delta d}$} & \multirow{2}{*}{$\frac{\delta s_{1 y}}{\delta d}$} & \multirow{2}{*}{$\frac{\delta s_{1}}{\delta d}$} \\
\hline (nrad) & (mas) & & & & & & & & & & & & \\
\hline 30 & 6 & 18.683 & 11.964 & 14.587 & -7.294 & -23.929 & 6.228 & 12.455 & 22.180 & -3.497 & \multirow{4}{*}{0.729} & \multirow{4}{*}{0.271} & \multirow{4}{*}{0.458} \\
\hline 60 & 12 & 37.366 & 23.929 & 29.174 & -14.587 & -47.857 & 12.455 & 24.911 & 44.360 & -6.994 & & & \\
\hline 120 & 25 & 74.732 & 47.857 & 58.349 & -29.174 & -95.715 & 24.911 & 49.821 & 88.721 & -13.989 & & & \\
\hline 240 & 50 & 149.464 & 95.715 & 116.698 & -58.349 & -191.430 & 49.821 & 99.643 & 177.441 & -27.977 & & & \\
\hline
\end{tabular}

Table 4: AFP suite.

These specifications are based on paraxial theory and assume collimated output to the Image Slicer. Minor adjustments (not shown here) are made using ray trace software to minimize aberrations and account for the slightly convergent output.

\subsection{Optical Performance}

Image quality at the Image Slicer and Exit Pupil of the projector suite are summarized in Table 5 for the four angular slit widths.

\begin{tabular}{|c|c|c|c|c|c|c|c|c|c|c|c|c|c|}
\hline \multirow{3}{*}{\multicolumn{2}{|c|}{\begin{tabular}{|l|} 
Angular \\
Slit \\
Width
\end{tabular}}} & \multirow{2}{*}{\multicolumn{2}{|c|}{$\begin{array}{l}\text { Image Slicer } \\
\text { Spaxel Size }\end{array}$}} & \multirow{2}{*}{\multicolumn{2}{|c|}{$\begin{array}{l}\text { Exit Pupil } \\
\text { Diameter }\end{array}$}} & \multicolumn{8}{|c|}{ RMS Image Blur } \\
\hline & & & & & & \multicolumn{4}{|c|}{ Image Slicer } & \multicolumn{4}{|c|}{ Exit Pupil } \\
\hline & & \multirow{2}{*}{$\begin{array}{c}\text { Spectral } \\
\mathrm{mm}\end{array}$} & \multirow{2}{*}{$\begin{array}{c}\text { Spatial } \\
\mathrm{mm}\end{array}$} & \multirow{2}{*}{\begin{tabular}{|c|} 
Spectral \\
$\mathrm{mm}$
\end{tabular}} & \multirow{2}{*}{$\begin{array}{c}\text { Spatial } \\
\mathrm{mm}\end{array}$} & \multicolumn{2}{|c|}{ Spectral } & \multicolumn{2}{|c|}{ Spatial } & \multicolumn{2}{|c|}{ Spectral } & \multicolumn{2}{|c|}{ Spatial } \\
\hline nrad & mas & & & & & $\mu \mathrm{m}$ & & $\mu \mathrm{m}$ & & $\mu \mathrm{m}$ & \begin{tabular}{|c|} 
Exit \\
Pupil \\
Diameter \\
\end{tabular} & $\mu \mathrm{m}$ & \begin{tabular}{|c|} 
Exit \\
Pupil \\
Diameter \\
\end{tabular} \\
\hline 30 & 6 & \multirow{4}{*}{2} & & & 27 & 90.1 & & 75.1 & & & & 0.0904 & \\
\hline 60 & 12 & & & 1.52 & 3.054 & 90.5 & 0.0453 & 75.3 & 0.0753 & 0.193 & 0.00 & 0.295 & \\
\hline 120 & 25 & & & 3.054 & 6.108 & 91.3 & 0.0 & 75.8 & 0.0 & 0.605 & & 1.11 & \\
\hline 240 & 50 & & & 6.108 & 12.215 & 92.9 & 0.0465 & 76.8 & 0.0768 & 2.24 & 0.000367 & 4.38 & 0.000359 \\
\hline
\end{tabular}

Table 5: Image quality at the Image Slicer and Exit Pupil for the four angular slit widths.

For the image slicer, image blur is shown in units of $\mu \mathrm{m}$ and spaxels. For the exit pupil, it is shown in units of $\mu \mathrm{m}$ and exit pupil diameter.

\subsection{The Opto-Mechanical System}

A view of the opto-mechanical deployment system is shown in Figure 13. 


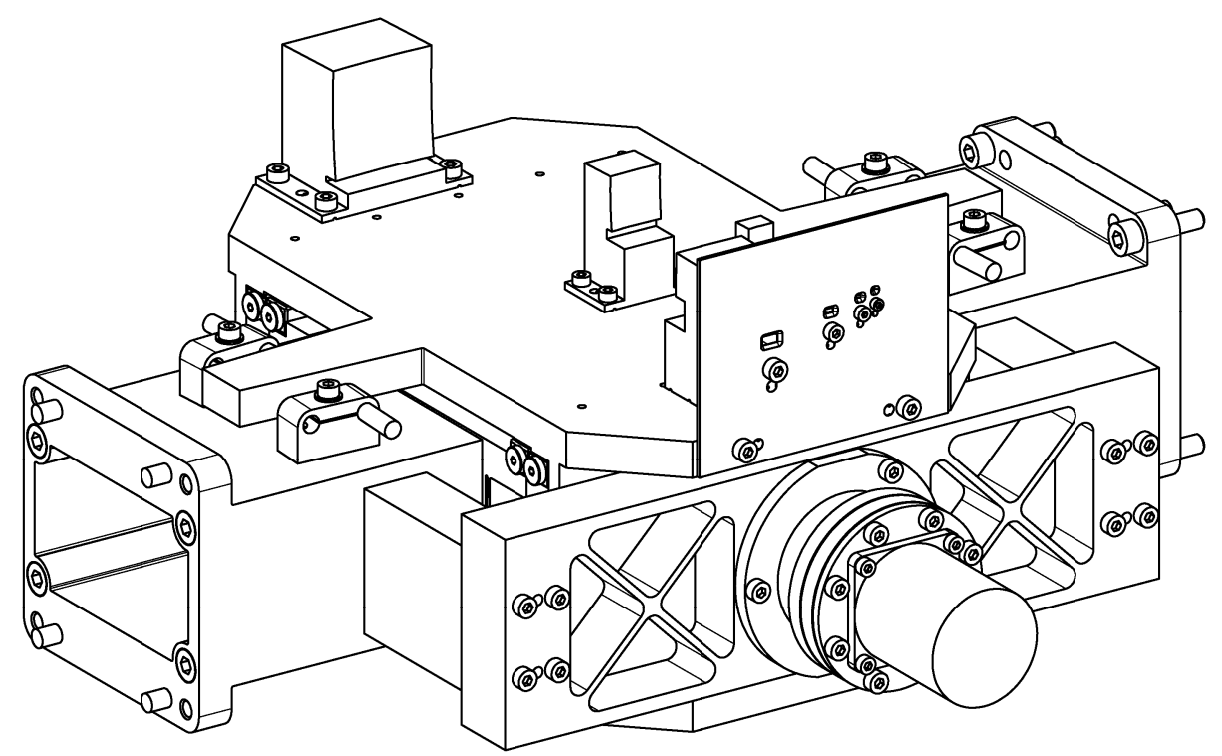

Figure 13: The opto-mechanical system for deployment of the four Anamorphic Projector Channels. Scale 2:5.

The mirrors take the form of posts mounted on the upper stage. This is translated on a linear bearing by means of a stepper motor drive to deploy the chosen channel. Position is determined to the required sub-micron level by means of eddy current sensors measuring the gap to a tapered target ramp. 